\title{
At-wavelength characterization of refractive x-ray lenses using a two-dimensional grating interferometer
}

\author{
Simon Rutishauser, ${ }^{1, a)}$ Irene Zanette, ${ }^{2}$ Timm Weitkamp, ${ }^{3}$ Tilman Donath, ${ }^{1,4}$ \\ and Christian David ${ }^{1}$ \\ ${ }^{1}$ Paul Scherrer Institut, 5232 Villigen PSI, Switzerland \\ ${ }^{2}$ European Synchrotron Radiation Facility, 38043 Grenoble, France \\ ${ }^{3}$ Synchrotron Soleil, 91192 Gif-sur-Yvette, France \\ ${ }^{4}$ Dectris Ltd., 5400 Baden, Switzerland
}

(Received 12 August 2011; accepted 10 November 2011; published online 29 November 2011)

\begin{abstract}
We report on the application of a two-dimensional hard x-ray grating interferometer to x-ray optics metrology. The interferometer is sensitive to refraction angles in two perpendicular directions with a precision of $10 \mathrm{nrad}$. It is used to observe the wavefront changes induced by a single parabolic beryllium focusing lens of large radius of curvature. The lens shape is reconstructed and its residual aberrations are analyzed. Its profile differs from an ideal parabolic shape by less than $2 \mu \mathrm{m}$ or $\lambda / 50$ at $\lambda=0.54 \AA$ wavelength. (C) 2011 American Institute of Physics. [doi:10.1063/1.3665063]
\end{abstract}

The performance of many experiments at current and future x-ray sources critically depends on a well defined, high quality wavefront. Ensuring this requires instruments to observe and quantify the performance of the x-ray optical elements and their effect on wavefront quality in-situ, at wavelength and under experimental conditions.

One such instrument is the one-dimensional hard x-ray grating interferometer, which has found widespread use in phase-contrast imaging, mainly of biological samples. ${ }^{1-4}$ It has also been applied to observe the wavefront downstream of grazing-incidence x-ray mirrors and monochromators. ${ }^{5-7}$ The one-dimensional grating interferometer is well suited for such tasks, as it provides a quantitative measurement of the differential wavefront phase in one direction with very high angular sensitivity, which is chosen to be along the optical element under test (i.e., in vertical direction for a vertically deflecting optical element).

In recent years, refractive x-ray optics such as parabolic compound refractive lenses have become increasingly important in many experiments at synchrotron sources. Unlike the simpler spherical $\mathrm{x}$-ray lenses, ${ }^{8}$ they show very weak spherical aberrations. They are widely used in microfocusing, ${ }^{9}$ full-field microscopy, ${ }^{10}$ for collimation ${ }^{11}$ and prefocusing, or as broadband monochromator. ${ }^{12}$ Due to their strongly curved surface, refractive x-ray lenses are extremely difficult to analyze by established metrology instruments such as contact profilometry or visible-light interferometry. In addition, defects in the bulk material of the lenses may affect their performance, and mere surface characterization methods are not sensitive to these effects. The use of penetrating radiation under conditions close or identical to the final use is therefore preferable for probing lens quality.

We report on quantitative measurements of the wavefront distortions introduced by a single parabolic beryllium focusing lens. The distortions were characterized using a two-dimensional grating interferometer. ${ }^{13}$ Unlike the onedimensional interferometer employed in earlier lens metrol-

\footnotetext{
a) Author to whom correspondence should be addressed. Electronic mail: simon.rutishauser@psi.ch.
}

ogy experiments with polychromatic $\mathrm{x}$-rays, ${ }^{15}$ the twodimensional grating interferometer allows for a simultaneous measurement of the full wavefront gradient vector downstream of the focusing lens and direct reconstruction of the lens profile and its aberrations. The use of monochromatic $\mathrm{x}$-rays enables a quantitative retrieval of the lens profile. The grating interferometer described here is well suited for measuring weakly focusing optical elements-it is thereby complementary to other phase retrieval methods such as the far-field diffraction based approach recently applied to a strongly focusing kinoform lens. ${ }^{14}$

Fig. 1 shows the measurement setup of the twodimensional grating interferometer, which is described in detail elsewhere. ${ }^{13}$ It consists of a checkerboard-pattern phase grating $G_{1}$ of period $p_{1}$ with $\pi$ phase shifting structures. The diffracted waves from this phase grating create a mesh-shaped interference pattern with half the period of $G_{1}$ at distances $d_{n}=n p_{1}^{2} /(8 \lambda)$ downstream, where $\lambda$ is the $\mathrm{x}$-ray wavelength and the Talbot order $n$ is an odd integer. As the interference pattern period is typically much smaller than the detector resolution, an absorbing analyzer grating $G_{2}$ with the same period $p_{2}$ as the interference pattern is introduced in the detector plane. The lateral position of the interference pattern at each detector pixel contains the phase information on the wavefront. To retrieve these positions, the grating is scanned in a two-dimensional raster scan along $x$ and $y$ over one or multiple periods of the interference pattern. This scan yields a two-dimensional oscillating signal at each pixel, which can be written as Fourier series:

$$
I\left(x_{\mathrm{g}}, y_{\mathrm{g}}\right)=\sum_{k, l} a_{k, l} \cos \left[\frac{2 \pi}{p_{2}}\left(k x_{\mathrm{g}}+l y_{\mathrm{g}}\right)+\varphi_{k, l}\right],
$$

where $x_{\mathrm{g}}$ and $y_{\mathrm{g}}$ are the raster scan position indices, $a_{k, l}$ are the Fourier coefficients, and $\varphi_{k, l}$ are their phases. In practice, the Fourier decomposition is implemented using a twodimensional fast Fourier transform.

The $\mathrm{x}$-ray transmission at each pixel is given by $T(u, v)=a_{0,0} / a_{0,0}^{\mathrm{r}}$, where the superscript ${ }^{\mathrm{r}}$ denotes a reference scan, recorded without the beryllium lens in the beam. 


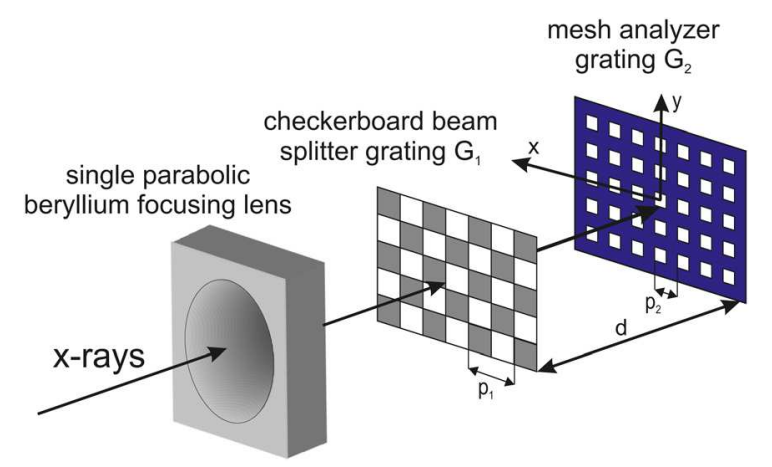

FIG. 1. (Color online) Two-dimensional grating interferometer setup with focusing lens under test. Downstream of the lens are the beam splitter phase grating $G_{1}$ with checkerboard structures of period $p_{1}$, the absorbing analyzer grating $G_{2}$ with mesh structures of period $p_{2}$, and the imaging detector downstream of $G_{2}$ (not depicted here).

For a two-dimensional raster scan over $N \times N$ grating periods, the changes in fringe phase $\Delta \varphi_{\mathrm{x}}=\varphi_{N, 0}-\varphi_{N, 0}^{\mathrm{r}}$ and $\Delta \varphi_{\mathrm{y}}=\varphi_{0, N}-\varphi_{0, N}^{\mathrm{r}}$ are proportional to the refraction angle in the sample $\alpha_{\mathrm{x}}=\Delta \varphi_{\mathrm{x}} p_{2} /(2 \pi d)$ along $x$ and equally along $y$. The focal length of the lens, equivalent to the radius of curvature of the wavefront, can be retrieved via the relation $f_{\mathrm{x}}=\left[\partial \alpha_{\mathrm{x}} / \partial x\right]^{-1}$.

Grating interferometry is not limited to measurements of single lenses but can also be applied to observing the wavefront of a compound beryllium refractive lens consisting of a stack of many single focusing lenses. If a reference scan is recorded in order to compensate for grating imperfections, there is a lower limit to the measurable focal length of $f_{\min }=\kappa d / p_{2}$, where $\kappa$ is the spatial resolution of the imaging system. This limitation is due to the fact that large refraction angles introduce wrapping of the measured differential phase, which can only be unambiguously unwrapped if $\mid \Delta \varphi_{\mathrm{x}}(i+1, j)$ $-\Delta \varphi_{\mathrm{x}}(i, j) \mid<\pi$. For even shorter focal lengths, $f_{\min }$ can be decreased by reducing the inter-grating distance $d$.

The experimental data shown here were recorded at the beamline ID19 of the European Synchrotron Radiation Facility. A photon energy of $23 \mathrm{keV}$ was selected using a Si-111 double crystal monochromator. The grating $G_{1}$ was positioned at $150 \mathrm{~m}$ from the wiggler source, with a distance of $\mathrm{d}=478 \mathrm{~mm}$ ( $n=9$ th Talbot order) to the absorption grating $G_{2}$. The checkerboard phase grating $G_{1}$ was realized by superimposing two silicon line gratings ${ }^{16}$ with period $p_{1}=4.785 \mu \mathrm{m}$ and orthogonal line orientation, each having a structure height $h_{1}=29 \mu \mathrm{m}$. The absorption grating $G_{2}$ was realized in a similar fashion by superimposing two gold line gratings ${ }^{17}$ of period $p_{2}=2.400 \mu \mathrm{m}$ and structure height $h_{2}=60 \mu \mathrm{m}$. The phase stepping raster scan was performed over one grating period in $8 \times 8$ steps. The images were recorded using a powder scintillator and a lens-coupled CCD camera with an effective pixel size of $7.5 \mu \mathrm{m}$, using an exposure time of $1 \mathrm{~s}$ per image. The minimum measurable focal length in this configuration is about $f_{\min } \approx 6 \mathrm{~m}$ assuming $30 \mu$ m spatial resolution. ${ }^{13}$

The tested lens was a single beryllium refractive lens with a profile of a paraboloid of revolution imprinted on the beryllium substrate from two sides. ${ }^{10}$ The nominal apex radius of curvature of the lens was $R=1 \mathrm{~mm}$, which at the photon energy used in our experiments corresponds to a
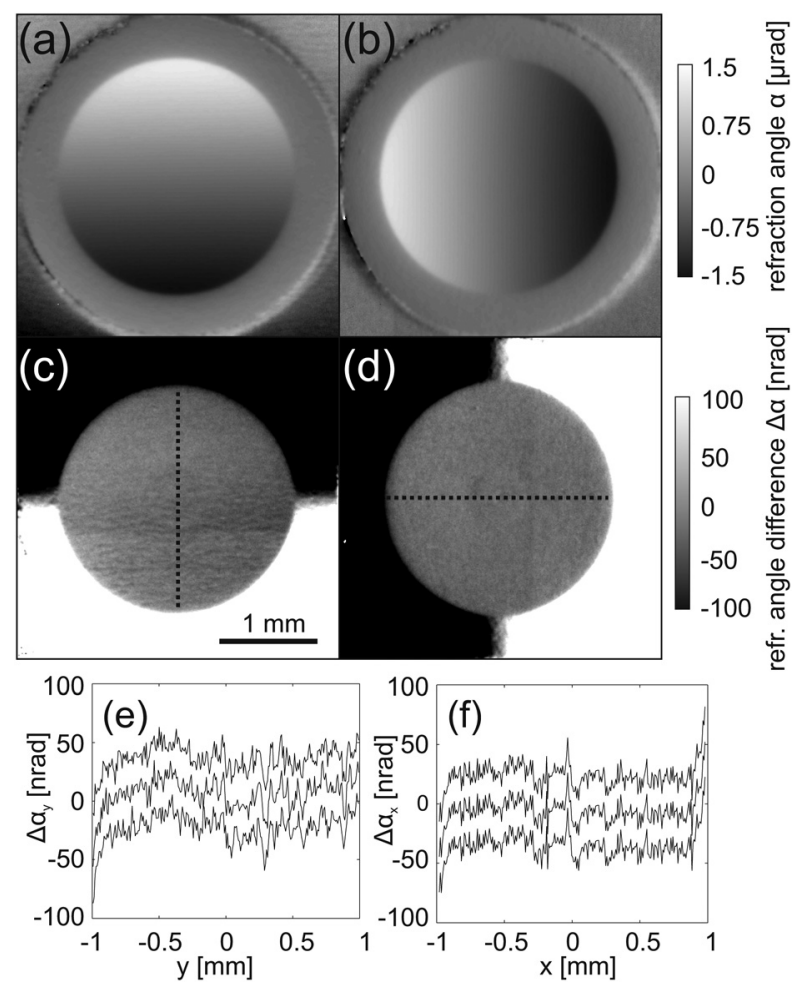

FIG. 2. Beryllium focusing lens with $\mathrm{R}=1 \mathrm{~mm}$ apex radius of curvature, measured using a two-dimensional grating interferometer. (a) and (b) Refraction angle $\alpha$ (proportional to the differential phase) of the beryllium focusing lens in vertical and horizontal direction. (c) and (d) Deviation in refraction angle $\Delta \alpha$ from the ideal lens shape. (e) and (f) Profiles from three adjacent lines offset in the plot by $30 \mathrm{nrad}$, at locations sketched in (c) and (d), respectively.

focal length of $f=R / 2 \delta=777 \mathrm{~m}$, where $\delta=6.437 \cdot 10^{-7}$ is the refractive index decrement. The diameter of its active area is $2.4 \mathrm{~mm}$. While in practice several dozen refractive lenses are often stacked to form a compound refractive lens of short focal length, the grating interferometer is highly sensitive and can be used to observe effects of a single refractive lens.

Figs. 2(a) and 2(b) shows a map of the refraction angle of the beryllium lens, measured both in vertical and horizontal direction. As expected for a parabolic lens, the refraction angle $\alpha$ changes linearly as a function of position in both directions. In order to observe residual aberrations more clearly, the difference to the ideal refraction angle $\Delta \alpha$ is shown in Figs. 2(c)-2(f). The standard deviation of $\Delta \alpha$ amounts only to $\sigma_{\Delta \alpha}=22 \mathrm{nrad}$. The precision (sensitivity) of the measurement, as determined from the standard deviation in a region of constant differential phase outside the lens, is 10 nrad.

The most evident deviation is observed at the lens center, where a circular shape with a diameter of about $0.4 \mathrm{~mm}$ is observed (Figs. 2(c) and 2(d)). Its origin can most likely be found in the fabrication of the tools used to imprint the lens profile in the beryllium substrate or in deformation of the tool in the imprint process, where the tool center is stressed the most.

From the refraction angle data in Figs. 2(a) and 2(b), which is proportional to the differential phase of the wavefront, the integrated wavefront phase can be reconstructed using a variety of algorithms. ${ }^{18-21}$ Some of these algorithms introduce low-frequency integration artifacts, though these 

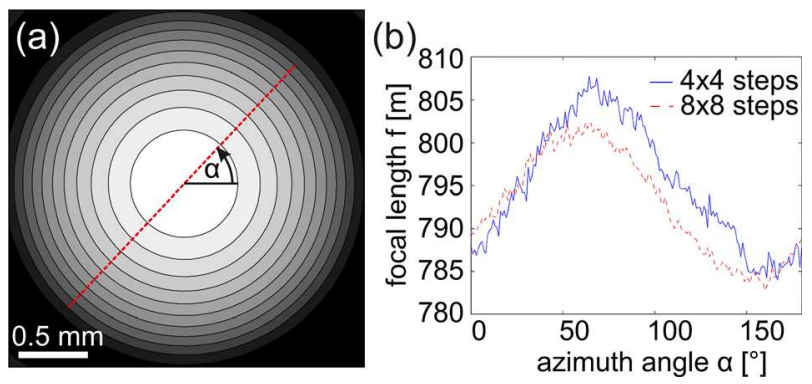

FIG. 3. (Color online) (a) Contour plot of the wavefront and beryllium lens profile reconstructed from Figs. 2(a) and 2(b), the lens profile changes by $100 \mu \mathrm{m}$ beryllium or $1.2 \lambda$ at $\lambda=0.54 \AA$ from one contour line to the next, (b) mean focal length along lines sketched in (a), plotted as function of azimuthal angle $\alpha$. The two curves are the results of two subsequent measurements, and they differ by $3 \mathrm{~m}$ on average. We observe a slight astigmatism of $\Delta f \mid f=2 \%$.

are small when using an $L_{1}$ norm optimization. ${ }^{21}$ The integrated wavefront phase, which is proportional to the beryllium lens profile, is shown as contour plot in Fig. 3. The mean difference of the measured lens profile to an ideal parabolic shape is less than $2 \mu \mathrm{m}$ or $\lambda / 50$ at the photon energy used for our measurements.

A decomposition of the reconstructed wavefront in Zernike polynomials ${ }^{22,23}$ yields small coefficients in the order of $1 \mu \mathrm{m}$ beryllium for all primary aberrations with exception of some residual coma $\left(Z_{n}^{m}=Z_{1}^{3}=4 \mu \mathrm{m}\right)$, spherical aberration $\left(Z_{0}^{4}=12 \mu \mathrm{m}\right)$, and astigmatism $\left(Z_{-2}^{2}=5 \mu \mathrm{m}, Z_{2}^{2}=10 \mu \mathrm{m}\right)$.

Fig. 3(b) shows the measured astigmatism in more detail. The mean focal length along profiles in different azimuthal directions through the lens center (along the dashed line sketched in Fig. 3(a)) is plotted-it varies slightly by up to $\Delta f / f=2 \%$. This information was extracted directly from linear combinations of Figs. 2(a) and 2(b), which yield the gradient vector in arbitrary directions and, based on its derivative, the focal length as well. The plot shows the focal length from two subsequent measurements of the same lens, using $4 \times 4$ and $8 \times 8$ raster scan steps, respectively, and the difference between the two measurements is less than 10 nrad in refraction angle or $3 \mathrm{~m}$ in terms of focal length. The measured astigmatism is so weak that it will usually be negligible in practical use of the lenses.

In independent measurements, it was verified that rotating a lens around its optical axis and comparing data recorded at several rotation angles does not decrease the reproducibility of the measurements. The interferometer does not show any measurable bias or distortion in any direction. An analog verification of the $L_{1}$ norm phase reconstruction shows that the algorithm introduces directional errors of less than $\lambda / 1000$.

In conclusion, we have demonstrated the application of two-dimensional hard $\mathrm{x}$-ray grating interferometry in optics metrology. The two-dimensional interferometer simultaneously provides the differential phase in two perpendicular directions with high sensitivity. From these data, the wavefront phase and the residual aberrations of a refractive beryllium lens have been retrieved, which is difficult using conventional profilometers. This method can also be applied to observing the wavefront of a compound refractive lens consisting of a stack of many single focusing lenses, or to any other optical element such as mirrors, monochromators, attenuators, or vacuum windows. Introducing moiré fringes by rotating the gratings with respect to each other, the interferometer can also be used for single shot measurements. ${ }^{5-7}$ While this reduces the spatial resolution, it enables independent retrieval of the wavefront phase for each recorded image.

The authors would like to thank E. Reznikova and J. Mohr, KIT/IMT for fabrication of the absorption gratings, A. Snigirev for supplying the beryllium lens, and M. GuizarSicairos for discussions. This work was carried out with the support of the Karlsruhe Nano Micro Facility (KNMF), a Helmholtz Research Infrastructure at Karlsruhe Institute of Technology (KIT). T.W. acknowledges support from RTRA "Digiteo" and RTRA "Triangle de la Physique."

${ }^{1}$ C. David, B. Nöhammer, H. Solak, and E. Ziegler, Appl. Phys. Lett. 81, 3287 (2002).

${ }^{2}$ A. Momose, S. Kawamoto, I. Koyama, and Y. Suzuki, Proc. SPIE 5535, 352 (2004).

${ }^{3}$ T. Weitkamp, A. Diaz, C. David, F. Pfeiffer, M. Stampanoni, P. Cloetens, and E. Ziegler, Opt. Express 13, 6296 (2005).

${ }^{4}$ G. Schulz, T. Weitkamp, I. Zanette, F. Pfeiffer, F. Beckmann, C. David, S. Rutishauser, E. Reznikova, and B. Müller, J. R. Soc., Interface 7, 1665 (2010).

${ }^{5}$ T. Weitkamp, B. Nöhammer, A. Diaz, C. David, and E. Ziegler, Appl. Phys. Lett. 86, 054101 (2005).

${ }^{6}$ E. Ziegler, L. Peverini, I. V. Kozhevnikov, T. Weitkamp, and C. David, AIP Conf. Proc. 879, 778 (2007).

${ }^{7}$ A. Diaz, C. Mocuta, J. Stangl, M. Keplinger, T. Weitkamp, F. Pfeiffer, C. David, T. H. Metzger, and G. Bauer, J. Synchrotron Radiat. 17, 299 (2010).

${ }^{8}$ A. Snigirev, V. Kohn, I. Snigireva, and B. Lengeler, Nature 384, 49 (1996).

${ }^{9}$ S. Bohic, A. Simionovici, A. Snigirev, R. Ortega, G. Devès, D. Heymann, and C. G. Schroer, Appl. Phys. Lett. 78, 3544 (2001).

${ }^{10}$ B. Lengeler, C. Schroer, J. Tümmler, B. Benner, M. Richwin, A. Snigirev, I. Snigireva, and M. Drakapoulos, J. Synchrotron Radiat. 6, 1153 (1999).

${ }^{11}$ A. I. Chumakov, R. Rffer, O. Leupold, A. Barla, H. Thiess, T. Asthalter, B. P. Doyle, A. Snigirev, and A. Q. R. Baron, Appl. Phys. Lett. 77, 31 (2000).

${ }^{12}$ G. Vaughan, J. Wright, A. Bytchkov, M. Rossat, H. Gleyzolle, I. Snigireva, and A. Snigirev, J. Synchrotron Radiat. 18, 125 (2011).

${ }^{13}$ I. Zanette, T. Weitkamp, T. Donath, S. Rutishauser, and C. David, Phys. Rev. Lett. 105, 248102 (2010).

${ }^{14}$ M. Guizar-Sicairos, S. Narayanan, A. Stein, M. Metzler, A. R. Sandy, J. R. Fienup, and K. Evans-Lutterodt, Appl. Phys. Lett. 98, 111108 (2011).

${ }^{15}$ M. Engelhardt, J. Baumann, M. Schuster, C. Kottler, F. Pfeiffer, O. Bunk, and C. David, Rev. Sci. Instrum. 78, 093707 (2007).

${ }^{16}$ C. David, J. Bruder, T. Rohbeck, C. Grünzweig, C. Kottler, A. Diaz, O. Bunk, and F. Pfeiffer, Microelectron. Eng. 84, 1172 (2007).

${ }^{17}$ E. Reznikova, J. Mohr, M. Boerner, V. Nazmov, and P.-J. Jakobs, Microsyst. Technol. 14, 1683 (2008).

${ }^{18}$ R. Frankot and R. Chellapa, IEEE Trans. Pattern Anal. Mach. Intell. 10, 439 (1988).

${ }^{19}$ A. Agrawal, R. Raskar, and R. Chellapa, in Computer Vision-ECCV 2006, 9th European Conference on Computer Vision, Lecture Notes in Computer Science, Vol. 3951 (Springer, Berlin, 2006), p. 578-591.

${ }^{20}$ C. Kottler, C. David, F. Pfeiffer, and O. Bunk, Opt. Express 15, 1175 (2007).

${ }^{21}$ Z. Fu, A. Robles-Kelly, and F. Lu, in 9th Biennial Conference of the Australian Pattern Recognition Society on Digital Image Computing Techniques and Applications (DICTA 2007), Glenelg, Australia (IEEE Computer Society, Washington, DC, 2007), p. 189.

${ }^{22}$ F. Zernike, Physica 1, 689 (1934).

${ }^{23} \mathrm{M}$. Born and E. Wolf, Principles of Optics (Cambridge University Press, Cambridge, 2002), p. 523. 